\title{
The solution of the prey and predator problem by differential transformation method
}

\author{
Belal Batiha \\ Higher Colleges of Technology (HCT), Abu Dhabi Men's College, United Arab Emirates (UAE) \\ E-mail: belalbatiha2002@yahoo.com, belal.batiha@hct.ac.ae
}

Copyright (C)2015 Belal Batiha. This is an open access article distributed under the Creative Commons Attribution License, which permits unrestricted use, distribution, and reproduction in any medium, provided the original work is properly cited.

\begin{abstract}
The problem of prey and predator is solved by the differential transformation method (DTM). Numerical comparisons with Adomian decomposition method (ADM) and power series method are presented.
\end{abstract}

Keywords: Differential transformation method; Taylor's series expansion; prey and predator problem; Adomian decomposition method.

\section{Introduction}

Almost all models of biological systems are essentially based on systems of non-linear ordinary differential equations (ODEs). Both mathematical modelling and simulation are very important in recent studies of biological mathematics. In this paper, we study the mathematical model of the prey-predator problem in which some rabbits and foxes are considered living together. Foxes eat the rabbits and rabbits eat clover. Suppose that there are enough clovers and the rabbits have enough food to eat. When there are a lot of rabbits, the foxes also grow and their population increase. When the number of foxes increase and they eat a lot of rabbits they enter into a short period of food and their number decrease. As the number of the foxes decrease, the rabbits will be safe and their population increase. When the number of rabbits increase the number of foxes would increase and by passing the time we can see an infinite repeatability of increase and decrease in the population of these two kinds of animals. The relationship of increasing and decreasing in the population of these two kind of animals can be described so-called mathematical model of the problem of prey-predator to the following system of non-linear equations:

$$
\begin{aligned}
& \frac{d x}{d t}=x(t)(a-b y(t)), \\
& \frac{d y}{d t}=-y(t)(c-d x(t)),
\end{aligned}
$$

where $x(t)$ and $y(t)$ are respectively the populations of rabbits and the foxes at the time $t$. The problem was solved by Biazar and Montazeri [1] using Adomian decomposition method (ADM), Biazar [2] using power series method, Rafei [3] by variation iteration method (VIM) and Goh [4] useing multistage variation iteration method (MVIM).

The motivation of this paper is to extend the analysis of the differential transformation method (DTM) to solve the prey and predator problem.

The concept of differential transformation method was first proposed by Zhou [5] in 1986 (see [6, 7, 8, 9]), and it was applied to solve linear and non-linear initial value problems in electric circuit analysis. This method constructs 
a semi-analytical numerical technique that uses Taylor series for the solution of differential equations in the form of a polynomial. It is different from the high-order Taylor series method which requires symbolic computation of the necessary derivatives of the data functions. The Taylor series method is computationally time-consuming especially for high order equations. The differential transform is an iterative procedure for obtaining analytic Taylor series solutions of differential equations. The Differential transformation method is very effective and powerful for solving various kinds of differential equations. For example, it was applied to two point boundary value problems [10], to differential-algebraic equations [11], to the $\mathrm{KdV}$ and $\mathrm{mKdV}$ equations [12], to the Schrödinger equations [13], to fractional differential equations [14] and to the Riccati differential equation [15]. Jang [16] introduced the application of the concept of the differential transformation of fixed grid size to approximate solutions of linear and non-linear initial value problems. Hassan [17] applied the differential transformation technique of fixed grid size to solve the higher-order initial value problems. The transformation method can be used to evaluate the approximating solution by the finite Taylor series and by an iteration procedure described by the transformed equations obtained from the original equation using the operations of differential transformation. The main advantage of this method is that it can be applied directly to linear and nonlinear ODEs without requiring linearization, discretization or perturbation. Another important advantage is that this method is capable of greatly reducing the size of computational work while still accurately providing the series solution with fast convergence rate [18].

In this paper, the total time evolution in the prey-predator problem is simulated by the numeric-analytic DTM. Numerical comparisons with the ADM and power series mthod are presented.

\section{The differential transformation method (DTM)}

An arbitrary function $f(x)$ can be expanded in Taylor series about a point $x=0$ as

$f(x)=\sum_{k=0}^{\infty} \frac{x^{k}}{k !}\left[\frac{d^{k} f}{d x^{k}}\right]_{x=0}$.

The differential transformation of $f(x)$ is defined as

$F(x)=\frac{1}{k !}\left[\frac{d^{k} f}{d x^{k}}\right]_{x=0}$.

Then the inverse differential transform is

$f(x)=\sum_{k=0}^{\infty} x^{k} F(k)$.

The fundamental mathematical operations performed by differential transform method are listed in Table 1 .

\subsection{The operation properties of differential transformation}

If $x(t), y(t)$ are two uncorrelated functions with time $t$ and $X(k), Y(k)$ are the transformed functions corresponding to $x(t), y(t)$ and the basic properties are shown as follows:

1. If $X(k)=D[x(t)], \quad Y(k)=D[y(t)]$, and $c_{1}$ and $c_{2}$ are independent of $t$ and $k$, then $D\left[c_{1} x(t)+c_{2} y(t)\right]=$ $c_{1} X(k)+c_{2} Y(k)$.

(Symbol $D$ denoting the differential transformation process).

2. If $z(t)=x(t) y(t), \quad x(t)=D^{-1}[X(k)], \quad y(t)=D^{-1}[Y(k)]$ and $\otimes$ denote the convolution, then

$$
D[z(t)]=D[x(t) y(t)]=X(k) \otimes Y(k)=\sum_{r=0}^{k} Y(r) X(k-r) .
$$

3. If $y(x)=y_{1}(x) y_{2}(x) \ldots y_{n-1}(x) y_{n}(x)$ then

$$
Y(k)=\sum_{k_{n-1}=0}^{k} \sum_{k_{n-2}=0}^{k_{n-1}} \ldots \sum_{k_{2}=0}^{k_{3}} \sum_{k_{1}=0}^{k_{2}} Y_{1}\left(k_{1}\right) Y_{2}\left(k_{2}-k_{1}\right) \ldots Y_{n-1}\left(k_{n-1}-k_{n-2}\right) Y_{n}\left(k-k_{n-1}\right) .
$$


Table 1: The fundamental operations of differential transformation method (DTM)

\begin{tabular}{|c|c|}
\hline Original function & Transformed function \\
\hline$y(x)=g(x) \pm h(x)$ & $Y(k)=G(k) \pm H(k)$ \\
\hline$y(x)=\alpha g(x)$ & $Y(k)=\alpha G(k)$ \\
\hline$y(x)=\frac{d g(x)}{d x}$ & $Y(k)=(k+1) G(k+1)$ \\
\hline$y(x)=\frac{d^{2} g(x)}{d x^{2}}$ & $Y(k)=(k+1)(k+2) G(k+2)$ \\
\hline$y(x)=\frac{d^{m} g(x)}{d x^{m}}$ & $Y(k)=(k+1)(k+2) \ldots(k+m) G(k+m)$ \\
\hline$y(x)=1$ & $Y(k)=\delta(k)$ \\
\hline$y(x)=x$ & $Y(k)=\delta(k-1)$ \\
\hline$y(x)=x^{m}$ & $Y(k)=\delta(k-m)=\left\{\begin{array}{ll}1, & k=m \\
0, & k \neq m\end{array}\right\}$ \\
\hline$y(x)=g(x) h(x)$ & $Y(k)=\sum_{m=0}^{k} H(m) G(k-m)$ \\
\hline$y(x)=e^{(\lambda x)}$ & $Y(k)=\frac{\lambda^{k}}{k !}$ \\
\hline$y(x)=(1+x)^{m}$ & $Y(k)=\frac{m(m-1) \ldots(m-k+1)}{k !}$ \\
\hline
\end{tabular}




\begin{tabular}{ccccccc}
\hline Case & $x_{0}$ & $y_{0}$ & $a$ & $b$ & $c$ & $d$ \\
\hline 1 & 14 & 18 & 1 & 1 & 0.1 & 1 \\
2 & 14 & 18 & 0.1 & 1 & 1 & 1 \\
3 & 16 & 10 & 0.1 & 1 & 1 & 1 \\
4 & 16 & 10 & 1 & 1 & 0.1 & 1 \\
\hline
\end{tabular}

\section{Application}

By using the fundamental operations of differential transformation method in Table 1, we obtained the following recurrence relation to the prey-predator problem:

$\begin{aligned} X(k+1) & =\frac{1}{(k+1)}\left[a X(k)-b \sum_{m=0}^{k} X(m) Y(k-m)\right], \\ Y(k+1) & =\frac{1}{(k+1)}\left[-c Y(k)+d \sum_{m=0}^{k} X(m) Y(k-m)\right] .\end{aligned}$

To make direct comparison with [1] and [2], we consider the following four cases:

\section{Case 1}

$a=1, b=1, c=0.1, d=1$ and the initial conditions $x(0)=14$ and $y(0)=18$.

From the initial condition $x(0)=14, y(0)=18$ we have $X(0)=14, \quad Y(0)=18$, and from equations $(5)$ and (6) we have

$X(0)=14, \quad X(1)=-238, \quad X(2)=271.6, \quad X(3)=20191.31333, \quad+\ldots$,

$Y(0)=18, \quad Y(1)=250.2, \quad Y(2)=-403.11, \quad Y(3)=-20087.343, \quad+\ldots$

Therefore, the closed form of the solution can be easily written as

$x(t)=\sum_{k=0}^{\infty} X(k) t^{k}=14-238 t+271.6 t^{2}+20191.31333 t^{3}+\ldots$,

$y(t)=\sum_{k=0}^{\infty} Y(k) t^{k}=18+250.2 t-403.11 t^{2}-20087.343 t^{3}+\ldots$

The above results are in complete agreement with the results obtained by the Adomian decomposition method $(\mathrm{ADM})$ and power series mthod reported in $[1,2]$.

\section{Case 2}

$a=0.1, b=1, c=1, d=1$ and the initial conditions $x(0)=14$ and $y(0)=18$.

From the initial condition $x(0)=14, y(0)=18$ we have $X(0)=14, \quad Y(0)=18$, and from equations $(5)$ and (6) we have

$X(0)=14, \quad X(1)=-250.6, \quad X(2)=604.87, \quad X(3)=19364.94233$,

$Y(0)=18, \quad Y(1)=234.0, \quad Y(2)=-734.40, \quad Y(3)=-19099.9800$,

Therefore, the closed form of the solution can be easily written as

$x(t)=\sum_{k=0}^{\infty} X(k) t^{k}=14-250.6 t+604.87 t^{2}+19364.94233 t^{3}+\ldots$,
$y(t)=\sum_{k=0}^{\infty} Y(k) t^{k}=18+234 t-734.4 t^{2}-19099.98 t^{3} \ldots$ 
The above results are in excellent agreement with the results obtained by the Adomian decomposition method $(\mathrm{ADM})$ and power series mthod reported in $[1,2]$.

\section{Case 3}

$a=0.1, b=1, c=1, d=1$ and the initial conditions $x(0)=16$ and $y(0)=10$.

From the initial condition $x(0)=16, y(0)=10$ we have $X(0)=16, \quad Y(0)=10$, and from equations $(5)$ and (6) we have

$$
\begin{gathered}
X(0)=16, \quad X(1)=-158.4, \quad X(2)=-415.92, \quad X(3)=7516.536, \\
Y(0)=10, \quad Y(1)=150.00, \quad Y(2)=333.00, \quad Y(3)=-7641.400,
\end{gathered}
$$

Therefore, the closed form of the solution can be easily written as

$$
\begin{aligned}
& x(t)=\sum_{k=0}^{\infty} X(k) t^{k}=16-158.4 t-415.92 t^{2}+7516.536 t^{3}+\ldots, \\
& y(t)=\sum_{k=0}^{\infty} Y(k) t^{k}=10+150 t+333 t^{2}-7641.4 t^{3}+\ldots
\end{aligned}
$$

The above results are in complete agreement with the results obtained by the Adomian decomposition method $(\mathrm{ADM})$ and power series mthod reported in $[1,2]$.

\section{Case 4}

$a=1, b=1, c=0.1, d=1$ and the initial conditions $x(0)=16$ and $y(0)=10$.

From the initial condition $x(0)=16, y(0)=10$ we have $X(0)=16, \quad Y(0)=10$, and from equations (5) and (6) we have

$X(0)=16, \quad X(1)=-144.0, \quad X(2)=-624.0, \quad X(3)=6602.40$,

$Y(0)=10, \quad Y(1)=159.00, \quad Y(2)=544.05, \quad Y(3)=-6828.535$,

Therefore, the closed form of the solution can be easily written as

$$
\begin{aligned}
& x(t)=\sum_{k=0}^{\infty} X(k) t^{k}=16-144 t-624 t^{2}+6602.4 t^{3}+\ldots, \\
& y(t)=\sum_{k=0}^{\infty} Y(k) t^{k}=10+159 t+544.05 t^{2}-6828.535 t^{3}+\ldots
\end{aligned}
$$

The above results are in excellent agreement with the results obtained by the Adomian decomposition method $(\mathrm{ADM})$ and power series mthod reported in $[1,2]$.

The following plots show the relations between the number of foxes and the rabbits versus time: 


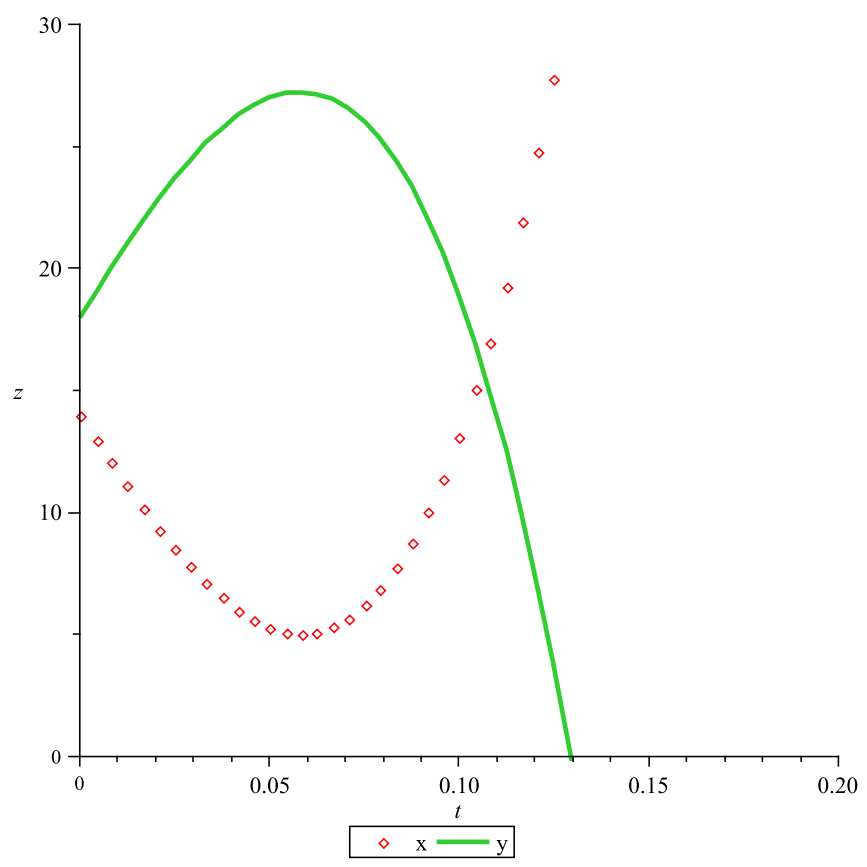

Figure 1: Number of rabbits and foxes versus time for Case 1

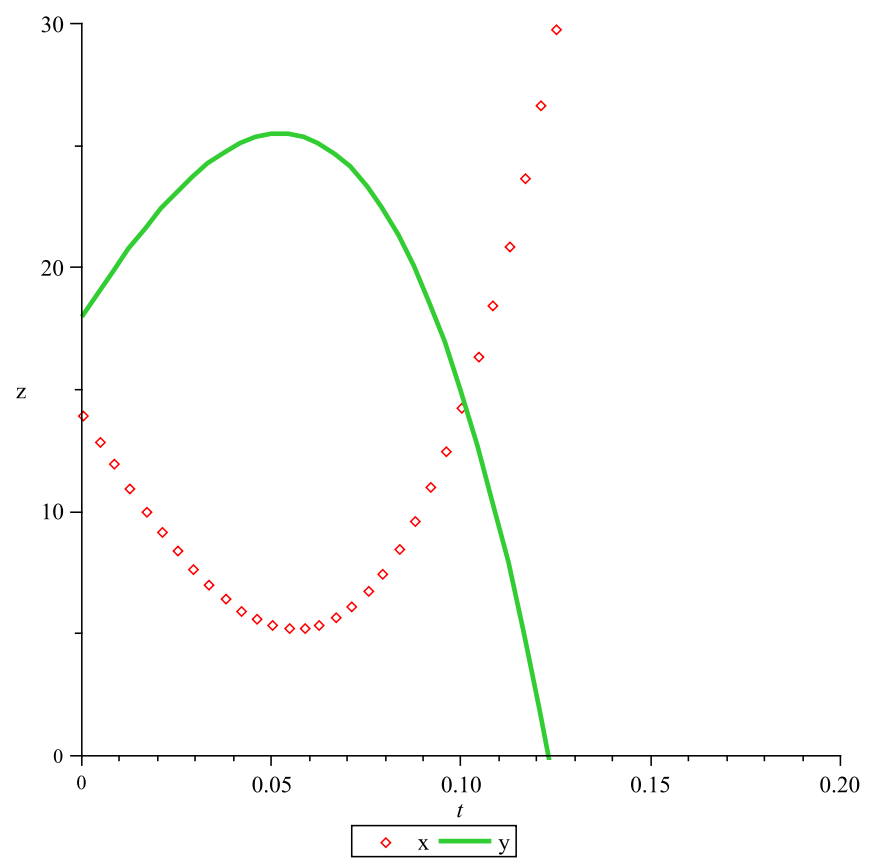

Figure 2: Number of rabbits and foxes versus time for Case 2

\section{Conclusions}

In this paper, a prey-predator problem was simulated accurately by the DTM. The method was used in a direct way without using linearization, perturbation or restrictive assumptions. Therefore, it is not affected by computation round off errors and one is not faced with necessity of large computer memory and time. There is less computation needed in comparison with the Adomian decomposition method. The results presented in this paper suggest that DTM is also readily applicable to prey-predator systems involving more populations. 


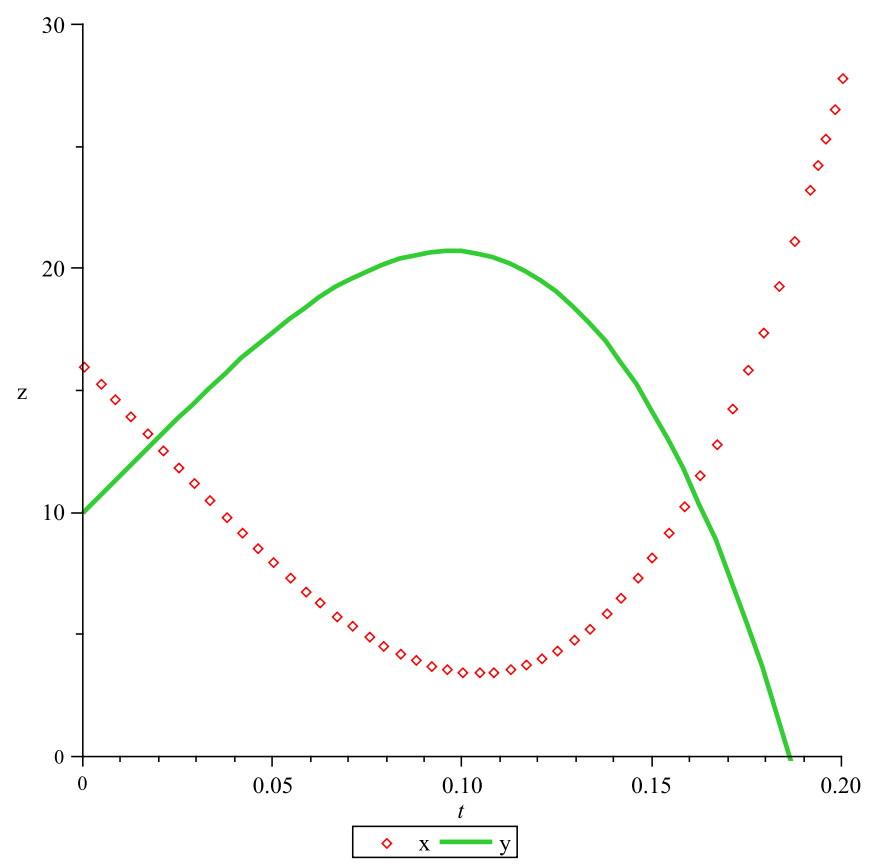

Figure 3: Number of rabbits and foxes versus time for Case 3

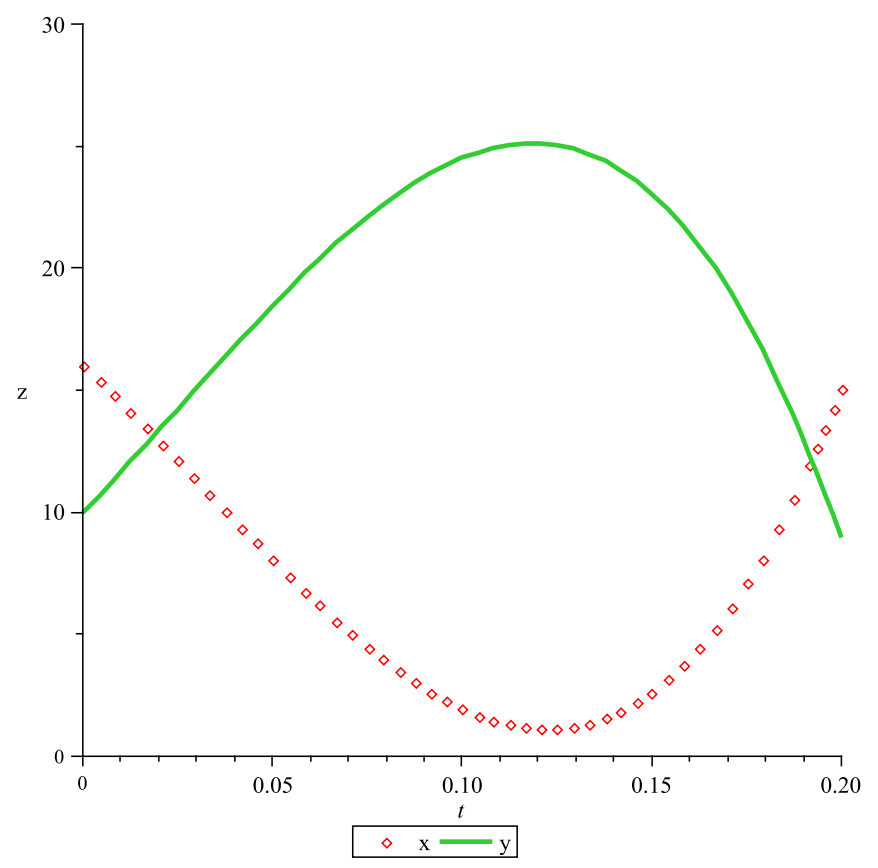

Figure 4: Number of rabbits and foxes versus time for Case 4

\section{References}

[1] J. Biazar, R. Montazeri, A computational method for solution of the prey and predator problem, Applied Mathematics and Computation 163 (2005) 841-847.

[2] J. Biazar, M. Ilie, A. Khoshkenar, A new approach to the solution of the prey and predator problem and comparison of the results with the Adomian method, Applied Mathematics and Computation 171 (2005) 486-491.

[3] M. Rafei, H. Daniali, D.D. Ganji, Variational iteration method for solving the epidemic model and the prey and predator problem, Applied Mathematics and Computation, 186 (2) (2007) 1701-1709. 
[4] S.M. Goh, M.S.M. Noorani, I. Hashim, Prescribing a multistage analytical method to a preypredator dynamical system, Physics Letters A, 373 (2008) 107-110.

[5] J.K. Zhou, Differential Transformation and Its Applications for Electrical Circuits, Huazhong University Press, Wuhan, China, 1986. (in Chinese).

[6] C.L. Chen, Y.C. Liu, Differential transformation technique for steady nonlinear heat conduction problems, Applied Mathematics and Computation 95 (1998) 155-164.

[7] C.L. Chen, Y.C. Liu, Solution of two point boundary value problems using the differential transformation method, Journal of Optimization Theory and Applications 99 (1998) 23-35.

[8] C.L. Chen, S.H. Lin, C.K. Chen, Application of Taylor transformation to nonlinear predictive control problem, Applied Mathematical Modeling 20 (1996) 699-710.

[9] C.K. Chen, S.H. Ho, Application of differential transformation to eigenvalue problems, Applied Mathematics and Computation 79 (1996) 173-188.

[10] C. L. Chen, Y. C. Liu. Solution of two point boundary value problems using the differential transformation method. J Opt Theory Appl., 99(1998):23-35.

[11] F. Ayaz. Applications of differential transform method to differential-algebraic equations. Applied Mathematics and Computation, 152(2004):649-657.

[12] F. Kangalgil, F. Ayaz. Solitary wave solutions for the KdV and mKdV equations by differential transform method. Chaos, Solitons and Fractals, 41(2009)(1):464-472.

[13] S. V. Ravi Kanth, K. Aruna. Two-dimensional differential transform method for solving linear and non-linear Schrödinger equations. Chaos, Solitons and Fractals, 41(2009)(5):2277-2281.

[14] A. Arikoglu, I. Ozkol. Solution of fractional differential equations by using differential transform method. Chaos, Solitons and Fractals, 34(2007):1473-1481.

[15] J. Biazar, M. Eslami, Differential Transform Method for Quadratic Riccati Differential Equation, International Journal of Nonlinear Science, 9 (4) (2010) 444-447.

[16] M.J. Jang, C.L. Chen, Y.C. Liy, On solving the initial value problems using the differential transformation method, Applied Mathematics and Computation 115 (2000) 145-160.

[17] I.H. A. Hassan, Differential transformation technique for solving higher-order initial value problems, Applied Mathematics and Computation 154 (2004) 299-311

[18] S-H. Chang, I-L. Chang, A new algorithm for calculating one-dimensional differential transform of nonlinear functions, Applied Mathematics and Computation 195 (2008) 799-808.

[19] S. Schnell,C. Mendoza, Closed form solution for time-dependent enzyme kinetics. J. Theor. Biol. 187 (1997) $207-212$.

[20] AK. Sen, An application of the Adomian decomposition method to the transient behavior of a model biochemical reaction. J. Math. Anal. Appl. 131 (1988) 232-245.

[21] I. Hashim, M.S.H. Chowdhury b, S. Mawa, On multistage homotopy-perturbation method applied to nonlinear biochemical reaction model, Chaos, Solitons and Fractals 36 (2008) 823-827. 